A N N A L E S Annales de Bretagne et des Pays de l'Ouest

Anjou. Maine. Poitou-Charente. Touraine

111-3 | 2004

Alcuin de York à Tours

\title{
De Tours à Rome : le corpus musical martinien au temps d'Alcuin
}

Jean-François Goudesenne

\section{OpenEdition}

Journals

Édition électronique

URL : http://journals.openedition.org/abpo/1259

DOI : $10.4000 /$ abpo.1259

ISBN : 978-2-7535-1495-9

ISSN : 2108-6443

Éditeur

Presses universitaires de Rennes

Édition imprimée

Date de publication : 20 septembre 2004

Pagination : 371-385

ISBN : 978-2-7535-0053-2

ISSN : 0399-0826

\section{Référence électronique}

Jean-François Goudesenne, "De Tours à Rome : le corpus musical martinien au temps d'Alcuin »,

Annales de Bretagne et des Pays de l'Ouest [En ligne], 111-3 | 2004, mis en ligne le 20 septembre 2006, consulté le 02 mai 2019. URL : http://journals.openedition.org/abpo/1259 ; DOI : 10.4000/abpo.1259 


\title{
De Tours à Rome : le corpus musical martinien au temps d'Alcuin
}

\author{
Jean-François GOUDESENNE \\ Institut de Recherche et d'Histoire des Textes (CNRS), Section de musicologie
}

\section{Les répertoires de chants pour saint Martin dans le monde latin ${ }^{1}$}

Deux travaux ont consacré une étude importante sur les répertoires de chant pour le grand confesseur du Iv siècle : Dom Guy Oury, qui après une première étude de la liturgie de la messe a rédigé en 1967 un article sur les formulaires et les différentes messes qui ont circulé dans le monde latin ${ }^{2}$. Ensuite, une étude non moins riche et documentée du Père Gy en 1988, concernant les répons de l'Office. Voici donc l'occasion d'établir avec quelques travaux complémentaires parus depuis, une mise en perspective et une synthèse sur le corpus des chants " martiniens " antérieur au milieu du $x^{\mathrm{e}}$ siècle.

Le sujet est bien ample, dans la mesure où saint Martin est loin de ne représenter, à la différence de tant d'autres, qu'un saint local et même qu'un grand patron des Gaules, fut-il emblématique pour la monarchie franque. Non seulement l'ancienneté du développement de son culte, qui renvoie vers les $\mathrm{V}^{\mathrm{e}}$ et $\mathrm{VI}^{\mathrm{e}}$ siècles (sa présence dans les mosaïques de Ravenne en est un illustre exemple), mais aussi l'ampleur de sa diffusion dans l'Église latine, en font un saint quasiment universel, qui dépasse de loin les Gaules et Rome. Cet état des choses nous invite donc à appréhender toute prospection dans le corpus liturgique et musical martinien en prenant compte l'ensemble des répertoires latins en usage avant et après l'unification romano-franque promue par les monarques carolingiens aux VIII et IX ${ }^{\mathrm{e}}$ siècles. Aussi, évitons de considérer le foyer de composition comme étant unique et "réservé " à Tours, ce haut lieu de pèlerinage, aussi prestigieux soit-il.

1. Sigles utilisés : GREG : répertoire romano-franc ou grégorien; ROM : répertoire vieuxromain, romain antique; MIL : répertoire ambrosien ou milanais.

2. "Les Messes de saint Martin... " Cet article fut suivi d'un autre dans le même périodique " Formulaires anciens..." . 
C'est justement cette constatation qui explique que les différentes traditions liturgiques qui ont été maintenues malgré les réformes carolingiennes, présentent pour la messe (dont l'établissement est plus ancien que l'office en général) des répertoires indépendants, qui ne sont pas ou peu en contact les uns avec les autres. Malgré leur rattachement partiel à la tradition hagiographique de Sulpice Sévère, il n'y a pas de point de contact entre les répertoires romano-franc et ambrosien, de même qu'entre romano-franc et le bénéventain. Ce dernier n'a par ailleurs que peu de points de contacts avec Milan, ce qui selon Thomas F. Kelly, est le signe d'une antériorité de Bénévent, qui a adapté des mélodies originales sur les textes hagiographiques du $\mathrm{v}^{\mathrm{e}}$ siècle $^{3}$. La tradition ambrosienne n'a pas non plus fait d'emprunt musical au grégorien, mais a également établi un répertoire propre à partir de quelques-uns des textes historiques de Sulpice Sévère, se référant un peu plus aux textes psalmiques, à l'instar de la liturgie hispanique avec laquelle Milan présente quelques points de contact ${ }^{4}$. La tradition mozarabe est quant à elle totalement indépendante des autres liturgies latines ${ }^{5}$.

\section{Les formulaires de la messe}

La fête de saint Martin ne se trouve pas toujours mentionnée dans les six antiphonaires de la messe retenus par Dom R.-J. Hesbert. La fête de saint Mennas, célébrée le même jour est plus ancienne et il faut considérer qu'une messe propre pour saint Martin seul est effectivement postérieure à l'antiphonale missarum primitif ${ }^{6}$. Dans les répertoires italiques, romains, et romano-francs, les formulaires se partagent entre des chants du commun dans un premier temps, qui correspondent en quelque sorte à un " archétype ", et des chants propres, qui remontent aux IX ${ }^{\mathrm{e}}-\mathrm{X}^{\mathrm{e}}$ siècles. Pour les messes du commun, les plus anciens graduels (fin VIII ${ }^{\mathrm{e}}-\mathrm{IX}^{\mathrm{e}}$ siècle) n'attribuent qu'une seule pièce à saint Martin, dont la fête est célébrée avec celle de Mennas. Il s'agit du répons-graduel Inveni David. Du reste, les formulaires donnent par la suite un commun des confesseurs, avec des options très variables ${ }^{7}$. Saint Martin ne reçoit pas de messe dans le vieuxromain, à l'exception d'un seul témoin ${ }^{8}$ : la fête n'est pas romaine et est bien introduite tardivement à Rome, dans le courant du VIII ${ }^{\mathrm{e}}$ siècle.

Beaucoup plus intéressantes, les messes propres se partagent en Europe entre deux formulaires : la plus répandue est la messe $O$ beatum virum, qui résulte d'une adaptation sur des pièces du commun des confesseurs du corpus hagiographique de Sulpice Sévère et de Grégoire de

3. The Beneventan Chant, c. "Benevento and the music of other liturgies ", p. 165 et p. $167-168$.

4. D. G. OURY, «Formulaires anciens... », p. 39.

5. Ibid., p. 38.

6. D. R.-J. HESBERT, Antiphonale missarum sextuplex, p. CIX-CX.

7. I. Sacerdotes tui; RG. Inveni David; All. Disposui; Off. Inveni David.

8. Arch. Cap. S. Pietri, F 22. 
De Tours à Rome : le corpus musical martinien au temps d'Alcuin

Tours $^{9}$. On la trouve en Aquitaine, en Lombardie ${ }^{10}$, en Francie occidentale ${ }^{11}$, en Angleterre ${ }^{12}$ et dans les livres franco-castillans ${ }^{13}$.

\begin{tabular}{|l|l|l|l|}
\hline & MEsse O Beatum virum & mode & Modèle (Graduel) \\
\hline Intrö̈t & O beatum virum in cuius & 3 & Cognovi (Sabine) \\
\hline Verset « tropé " & $\begin{array}{l}\text { Ecclesia virtutum } \\
\text { roboratur }\end{array}$ & & $\begin{array}{l}\text { Constitues eos } \\
\text { (Pierre et Paul) }\end{array}$ \\
\hline Graduel & Ora pro nobis & 6 & $\begin{array}{l}\text { In die solemnitatis } \\
\text { (Fer. v Paschae) }\end{array}$ \\
\hline Alleluia & V. Dum sacramenta & & Oculis ac manibus \\
\hline Offertoire & Martinus igitur & 1 & Erubescant (temporal) \\
\hline
\end{tabular}

Le second formulaire correspond à la messe Beatus Martinus qui, de façon plus visible encore que la précédente, se présente comme une adaptation d'une messe du commun d'un confesseur. Sa diffusion est restreinte à l'Italie $^{15}$. En plus de ces deux messes, s'ajoutent de nombreuses pièces isolées et locales, notamment des alleluias ${ }^{16}$, puis des compositions locales, notamment à Forlimpopoli (XI ${ }^{\mathrm{e}}-\mathrm{XII}{ }^{\mathrm{e}}$ siècles) et au Mont-Cassin (1 ${ }^{\text {re }}$ moitié XII ${ }^{\mathrm{e}}$ siècle) ${ }^{17}$.

\begin{tabular}{|l|l|l|l|}
\hline & $\begin{array}{l}\text { MESSE « Beatus } \\
\text { Martinus " }\end{array}$ & mode & Modèle (Graduel) \\
\hline Introït & Beatus Martinus obitum & 7 & $\begin{array}{l}\text { In virtute } \\
\text { (Valentin, Théodore...) }\end{array}$ \\
\hline Graduel & Dixerunt discipuli & 6 & $\begin{array}{l}\text { Beatus vir } \\
\text { (Valentin, Matthieu) }\end{array}$ \\
\hline & V. Invadent enim & & \\
\hline
\end{tabular}

9. Sources des textes (messe comparée à l'office) : I = R. O beatum virum ( $8^{\mathrm{e}}$ de l'office) ; Texte du trope = Verset du R. O beatum virum (ecclesia virtute... quem Michael... cum angelis) ou corps du R. Ecclesia virtute $\left(12^{\mathrm{e}} \mathrm{R}\right)>$ Grégoire de Tours, Des vertus de s. Martin (BHL 5618), livre I, c. 5; voir aussi Pseudo-Alcuin, Sermo de transitu, c. 15, 5 et ss. Enfin voir Remi, A. Gloriosus cultor, verset : laetificavit angelos demones terruit, ecclesiam meritis et virtutibus roboravit. $\mathrm{G}=\mathrm{R}$ commun 1 ?; $\mathrm{V}=$ Verset du $\mathrm{R} 4$ ou $\mathrm{R} 5 ; \mathrm{All}=\mathrm{R} 4 ; \mathrm{O}$ = R6; $\mathrm{V}=$ Verset $\mathrm{R} 6 ; \mathrm{C}=\mathrm{R} 9$.

10. Brescia (Oxford, Bodleian Library, Lit. 366, $\left.\mathrm{f}^{\circ} 3211^{\mathrm{e}} \mathrm{s} . \mathrm{m}\right)$ (sans notation); Bergamo, B. Civica, MA $150, \mathrm{f}^{\circ} 100 \mathrm{v}^{\circ}$, fin $\mathrm{XI}^{\mathrm{e}}$ siècle /début XII ${ }^{\mathrm{e}}$ siècle, noté sur lignes avec pointe sèche.

11. Auxerre-Rouen-Paris-Vendôme.

12. Oxford, Bodleian Library Rawlinson 892.

13. D. G. OuRY, "Formulaires anciens... ", p. 30-31.

14. Cet offertoire en $1^{\mathrm{er}}$ mode est atypique et semble présenter une facture "gallicane".

15. Bénévent, Vérone, Pavie, Bobbio, Mont-Cassin etc. Mais des pièces pourraient avoir une provenance et une diffusion dans les Gaules, par exemple l'offertoire de $2^{\mathrm{e}}$ mode atypique ou encore l'Alleluia avec prosule et séquence, voir O. MARCUSSON, Prosules de la Messe..., n ${ }^{\circ} 36,8$.

16. Voir D. G. OuRY, "Formulaires anciens... ", p. 33-34.

17. Of. Veniens miles ad M. ; C. Cum venisset (> Sulp. Sév., c. 16). 


\begin{tabular}{|l|l|l|l|}
\hline Alleluia & Oculis ac manibus & 2 & $\begin{array}{l}\text { Type 'Dies sanctificatus' } \\
\text { (Nativ.) }\end{array}$ \\
\hline & $\begin{array}{l}\text { Beatus vir sanctus } \\
\text { Martinus }\end{array}$ & 7 & \\
\hline Offertoire $^{18}$ & O virum ineffabilem & 2 & Off. gallicans \\
\hline & V. O beatum virum & & $\begin{array}{l}\text { Posuisti } \\
\text { (nombreux saints) }\end{array}$ \\
\hline Communion & Sacerdos dei Martine & 6 & 6 \\
\hline
\end{tabular}

\section{L'Office}

Pour l'office, les choses semblent un peu plus complexes. Si les textes de la messe sont parfois communs avec ceux de l'Office, il s'agit bien de compositions totalement indépendantes, et certainement d'époques différentes, l'office s'imposant par son antériorité ${ }^{19}$. Seule la tradition ambrosienne présente une série d'antiennes historiques ${ }^{20}$ correspondant à GREG et à ROM pour quelques pièces (voir infra).

Il convient donc de bien distinguer dans les antiphonaires et les bréviaires grégoriens les plus courants les strates, correspondant à diverses campagnes de composition, par la suite unifiées dans le cursus monastique $\mathrm{au} \mathrm{x}^{\mathrm{e}}$ siècle et réparties pour diverses fêtes. Ainsi, parmi les nombreuses antiennes, on peut dégager une série primitive, une série additionnelle et surtout celles composées par Odon de Cluny (927-942) pour la fête de l'octave. De même, les répons ne sauraient être considérés comme d'un seul bloc - certes dans une moindre mesure que les antiennes.

Les répertoires grégoriens sembleraient donc plus tardifs que les bénéventain, ambrosien et hispanique, surtout pour la messe, mais aussi pour l'office, en particulier la série de répons, probablement plus tardive que les antiennes, composées dans une campagne antérieure. Cette étude comparative des rites permet d'établir un terminus a quo, lui-même dépendant d'hypothèses peu sûres quant à une datation précise des répertoires ambrosiens et bénéventains.

\section{Le formulaire de Tours présent dans l'antiphonaire vieux-romain}

La présence d'un office de saint Martin dans le répertoire vieux-romain est tout à fait exceptionnelle par rapport aux autres répertoires latins, dans la mesure où son formulaire est le seul à correspondre à celui des livres romano-francs. Pour Dom Morin, le développement du culte de saint Martin

18. Cet offertoire en $1^{\text {er }}$ mode est atypique et semble également présenter une facture "gallicane".

19. Lorsque les textes correspondent (on pourrait envisager parfois un emprunt des textes de la messe à ceux de l'Office), les modes de l'office grégorien ne correspondent aucunement aux pièces de la messe, en haute Italie ou ailleurs (messe $O$ beatum virum).

20. Textes hagiographiques de Sulpice Sévère. 
en Italie s'effectue à l'époque des guerres gothiques ( $\mathrm{VI}^{\mathrm{e}}$ siècle) et l'introduction de sa fête à Rome est probablement liée à la fondation d'une basilique intra muros au $\mathrm{VI}^{\mathrm{e}}$ siècle par le pape Symmaque (498-514) au titulum de saint Sylvestre et d'Equitius, devenu par la suite saint Martin ai monti ${ }^{21}$. Certes, le développement d'un culte et l'instauration d'une fête est une chose, mais la dotation d'un office liturgique propre en est une autre : elle implique un plus haut degré de développement de ce culte. Antoine Chavasse constate comme Dom Morin puis Dom R.-J. Hesbert une instauration tardive à Rome de la fête de saint Martin dans la liturgie ${ }^{22}$ ce qui rejoint finalement l'ancien point de vue de Gastoué selon lequel l'office de saint Martin pourrait être considéré comme une addition non romaine de la fin du $\mathrm{VIII}^{\mathrm{e}}$ siècle, à l'imitation des répertoires grégoriens, intégrés à des éléments locaux préexistants ${ }^{23}$. Deux témoins donnent donc l'office : les deux antiphonaires de Saint-Pierre de Rome, Vaticano, Archivio di San Pietro B 79, $\mathrm{f}^{\circ}$ 168-169 v ${ }^{24}$; London, Bristish Library, Add. 29988, f ${ }^{\circ}$ 137-139 (pl. 1).

\section{Correspondances du formulaire vieux-romain et du grégorien}

Le formulaire romain de l'office correspond globalement et approximativement tant dans son corpus que dans l'ordre du formulaire et dans le profil de ses mélodies, au formulaire grégorien. Il est un peu moins développé néanmoins, ne donnant que huit répons de matines et les seules antiennes de laudes. Certes, le degré de similitude des mélodies varie, de l'analogie assez évidente, à la transposition et la réécriture, surtout pour les pièces en deutérus, " adaptées " en tétrardus dans l'usage romain, plus fleuri, plus orné, mais beaucoup plus stéréotypé et moins diversifié que le grégorien, tant au niveau de la centonisation que du répertoire de formules et la répartition différenciée des modes.

\begin{tabular}{|c|c|c|}
\hline London, B. L. Add. 29188 & Vaticano, Arch. San Pietro B 79 & GREG \\
\hline \multicolumn{3}{|l|}{ RESPONSORIA } \\
\hline $\begin{array}{l}\text { Beatus M. electus } \\
\text { V. Sancte Trinitatis }\end{array}$ & " V. Dum sacramenta & $\begin{array}{l}\text { Hic est Martinus } \\
=\end{array}$ \\
\hline $\begin{array}{l}\text { Ora pro nobis } \\
\text { V. Ut digni }\end{array}$ & $"$ & " mode \# \\
\hline $\begin{array}{l}\text { Dum sacramenta } \\
\text { V. Oculis }\end{array}$ & $"$ & " mode \# \\
\hline $\begin{array}{l}\text { Martinus abrahe } \\
\text { V. Oculis }\end{array}$ & "V. Dum sacramenta & " mode \# \\
\hline $\begin{array}{l}\text { O beatum virum M. antistitem } \\
\text { V. Domine si adhuc }\end{array}$ & " & $\begin{array}{l}" \\
=\end{array}$ \\
\hline
\end{tabular}

21. D. G. Morin, « Liturgie et basiliques de Rome... ", p. 316, n. 7. C. Pietri puis P. Bernard considèrent qu'il s'agissait de deux basiliques distinctes, voir P. BERNARD, Du chant romain au grégorien, p. 297, n. 7.

22. A. ChavASSE, La liturgie de la ville de Rome..., p. 171.

23. A. GASTOUE, Les origines du chant romain..., p. 279.

24. Édition fac-similé de G. BAROFFIO et S. J. Kim, Biblioteca apostolica Vaticana Archivio S. Pietro B 79, Antifonario della basilica di S. Pietro (sec. XII), Torre d'Orfeo, Roma, 1995. 


\begin{tabular}{|c|c|c|}
\hline $\begin{array}{l}\text { Dixerunt } \\
\text { V. Scimus }\end{array}$ & " & $\begin{array}{l}\text { " } \\
=\end{array}$ \\
\hline $\begin{array}{l}\text { Beatus vir in cuius transitu } \\
\text { V. Ecclesia virtute }\end{array}$ & " & I \\
\hline $\begin{array}{l}\text { Beatus Martinus obitum } \\
\text { V. Respondens }\end{array}$ & & $\begin{array}{l}\text { " } \\
=\end{array}$ \\
\hline $\begin{array}{l}\text { Ecce magnum } \\
\text { V. Dispersit }\end{array}$ & " & / \\
\hline \multicolumn{3}{|l|}{ ANTIPHONAE } \\
\hline Martine misit & " & $=$ \\
\hline Martinus adhuc & " & $=$ \\
\hline Beatus Martinus dixit & " & \# \\
\hline Martinus abrahe & " & $=$ \\
\hline Dum sacramenta & " & $=$ \\
\hline Dixerunt & " & $=$ \\
\hline Sanctus Martinus suam cecidit & & I \\
\hline Inposita manu & & $?$ \\
\hline O quantus luctus & " & $\#$ \\
\hline
\end{tabular}

\section{La musique : formes, structures et modèles}

Les répons

Hic est Martinus : Bien que consideré par Walter Howard Frere comme inférieur à d'autres répons plus anciens ${ }^{25}$, ce répons appartient néanmoins à un groupe très homogène du tétrardus, dans lequel on retrouve les répons Spiritus sanctus replevit de la Pentecôte et Hic est fratrum pour les livres historiques de la Bible, en l'occurrence les Macchabées. Tous ces répons ont exactement les mêmes enchaînements de formules.

Les deux répons Dixerunt discipuli* et Domine si adhuc présentent le groupe le plus ancien du second mode, auquel ils se conforment moins strictement; mais ils présentent les caractéristiques du vieux-fonds ${ }^{26}$. D'ailleurs on le retrouve tel quel dans $\mathrm{ROM}^{*}$. De même le répons Martinus abrahae du premier mode est tout à fait conforme au groupe dominant et le plus stable ${ }^{27}$. Mais ROM l'a transposé en huitième mode.

Les deux répons $O$ beatum virum semblent également avoir été composés ensemble, d'autant plus qu'ils se retrouvent dans le même mode et avec la même mélodie dans ROM. Walter Howard Frere les rattache au VIII ${ }^{a}$, premier groupe, le plus stable comme le $I^{a}$ et correspondant au ROM ${ }^{28}$. Suivant le même enchaînement de formules, on serait tenté de considérer le répons Hic est Michael comme leur modèle, les offices ajoutés aux VIII $\mathrm{e}_{-}$ $\mathrm{IX}^{\mathrm{e}}$ siècles prenant parfois modèle sur des fêtes proches dans le temps liturgique (voir infra).

25. W. H. FRERE, Antiphonale Sarisburiense..., p. 44.

26. Ibid., p. 8.

27. Ibid., p. 18.

28. Ibid., p. 52. 
Le répons Dum sacramenta fait également partie d'un groupe du vieuxfonds, le $I V^{a}$. Walter Howard Frere rattache à ce répons le célèbre répons Diffusa est gratia du Commun des Vierges ainsi que des répons d'offices plus tardifs tels saint Sébastien, la décollation de Jean-Baptiste ou d'autres fêtes mariales (Purification) ${ }^{29}$.

Enfin, le répons $O$ quantus erat, utilisant des matériaux du même groupe $^{30}$ se distingue du noyau primitif de l'office (il n'est pas dans ROM d'ailleurs) est se présente manifestement comme une adaptation de répons du commun, notamment Miles Christi - ce qui n'a rien de surprenant dans la rhétorique martinienne des hagiographes.

L'office de saint Martin ne se situe pas dans son cycle de répons, du moins pour le noyau primitif, parmi les offices ajoutés au début du $\mathrm{IX}^{\mathrm{e}}$ siècle, par exemple saint Denis ${ }^{31}$. Il semblerait se situer entre les Historiae de l'Ancien Testament et les offices ajoutés au sanctoral romain du VIII ${ }^{\mathrm{e}}$ siècle comme Sébastien ou Hyppolitte ${ }^{32}$. Modelé sur des pièces du vieux-fonds, notamment l'office de l'Archange, rien ne s'opposerait à ce que son profil stylistique remonte effectivement au milieu du VIII ${ }^{\mathrm{e}}$ siècle.

Les antiennes quant à elles pourraient très bien remonter à une campagne de composition antérieure, tant leur profil correspond aux offices romains du vieux-fonds : composition en série dans un même mode, sur des timbres identiques ou apparentés, en l'occurrence le tétrardus. La brièveté des textes, qui ne laisse aucune place à la centonisation à l'instar d'offices du IX ${ }^{\mathrm{e}}$ siècle (saint Denis, saint Remi, saint Quentin), comme la pureté des timbres, ne laissent aucun doute sur l'ancienneté du cycle des antiennes de matines et des laudes. Une antienne en protus-quarte, Dum sacramenta, indique par ailleurs un archaïsme ${ }^{33}$. La correspondance très relative avec ROM, qui se manifeste certes au niveau du mode mais pas toujours à celui du timbre semble exclure un office d'origine romaine envoyé en Gaule avec un texte mélodique noté.

\section{Le témoignage d'un Pseudo-Alcuin et la genèse de l'office}

Le texte correspondant au premier répons Hic est Martinus (CAO 6825) se trouve entièrement cité dans le Sermo de transitu san Martini, attribué à Alcuin par Migne, à propos de la grâce apostolique ${ }^{34}$ représentée par le grand confesseur de l'Occident latin :

29. Ibid., p. 34 .

30. Ibid., p. 36.

31. J.-F. GOUDESENNE, L'Office romano-franc des saints martyrs Denis...

32. A. Gastoue, Les origines du chant romain..., p. 279 et R. Jonsson, Historia ...

33. Voir D. J. CLAIRE, "Les répertoires liturgiques latins avant l'octoechos... ", p. 126136 ; C. MAITRE, « Le protus quarte dans le répertoire ambrosien ", p. 517-527.

34. Voir aussi J. MABILLON, Annales, année 710. D'après C. Veyrard-Cosme, les références aux apôtres et ces formules littéraires sont des topos récurrents dans la correspondance d'Alcuin. 
" [...] egregius sacerdotum, imitator apostolorum et Christi praedicator. Quem dominus in gratia apostolica est dignatus eligere, sicut de illo scriptum est : Hic est Martinus electus dei pontifex, Cui dominus post apostolos tantam graciam conferre dignatus est, Ut in virtute trinitatis deifice, Mereretur fieri trium mortuorum suscitator magnificus. Sancte trinitatis fidem Martinus confessus est ${ }^{35}$."

Alors que son attribution a été récemment remise en cause par Michael Gorman $^{36}$, ce Sermo que d'aucuns appellent également Laudatio sancti Martini ${ }^{37}$ constitue néanmoins un terminus ante quem solide pour dater la composition de la majeure partie de l'office, qui s'impose à l'évidence comme antérieure sinon contemporaine à l'abbatiat d'Alcuin à Tours.

En effet, les travaux de I. Deug Su, qui certes distingue le Sermo de la Vita écrite par Alcuin (BHL 5625) mais considère que ces deux ouvres hagiographiques ont été rédigées à la même époque, avant 800 . Ce texte hic est Martinus qui n'est peut-être pas celui du répons mais une source commune, serait une inscription gravée sur le vénérable tombeau du grand patron des Gaules ${ }^{38}$. (pl. 2)

Si Edmond Le Blant souligne l'importance des inscriptions de la basilique de Tours des $\mathrm{V}^{\mathrm{e}}$ et $\mathrm{vl}^{\mathrm{e}}$ siècles qui eurent une célébrité immense et s'il constate l'origine liturgique de certaines ${ }^{39}$, nos recherches du côté de l'épigraphie n'ont pas vraiment abouti, que ce soit celle du tombeau, de la cellule ou de la basilique, ses autels, son abside. En effet, parmi les inscriptions que l'on trouve dans les martinelli, qui s'imposent comme les témoins archéologiques les plus fiables compte tenu des incessantes destructions et reconstructions du tombeau entre le $\mathrm{VIII}^{\mathrm{e}}$ et le $\mathrm{XI}^{\mathrm{e}}$ siècle, les plus pertinentes sont les suivantes, gravées de part et d'autre du tombeau :

ITEM CIRCA TUMULUM AB UNO LATERE

Hic conditus est sanctae memoriae martinus episcopus.

Cuius anima in manu dei est

Sed hic totus est praesens.

Manifestus omni gratia virtutum

ITEM IN ALIO LATER

Certamen bonum certavit. Cursum consummavit. Fidem servavit : decetero reposita est illi corona iustitiae. Quam reddet illi dominus in illa die iustus iude ${ }^{40}$.

Le collections épigraphiques mérovingiennes, gravées dans l'ancienne basilique érigée par saint Perpet, décrite par Grégoire de Tours ${ }^{41}$, avec les

35. PL 101, col. 662.

36. M. GoRMAN, " Alcuin before Migne », p. 101-130.

37. J. LAHACHE, Biblioteca sanctorum, t. 8, col. 1248-1291.

38. I. Deug-Su, «L’Opera agiografica di Alcuino... », p. 57-83. Voir également M.-H. JuLLIEN et F. Perelman, Clavis...

39. Pages CVI-CVII, t. II, $\mathrm{n}^{\circ} 392$ : plusieurs des inscriptions de Tours semblent avoir été inspirées par les vieux offices du saint. La légende Certamen bonum certavit ( $\mathrm{n}^{\circ}{ }^{179)}$ reproduit un passage de l'épître à Timothée, lue lors de la fête de $\mathrm{s}$. Martin.

40. E. LE BLANT, Inscriptions chrétiennes de la Gaule...

41. Historia Francorum. 
contributions de Fortunat, Paulin de Périgueux ou Sidoine Apollinaire ${ }^{42}$, n'apporte aucun résultat, ce qui me laisse penser que s'il y a une source épigraphique, elle se situerait entre cette collection et d'autres plus tardives $^{43}$. Il pourrait s'agir éventuellement d'une nouvelle inscription générée par une campagne de renouvellement du culte occasionnée par diverses destructions (invasions sarrazines des années 730).

Nul doute que le style du texte lui-même avec ce démonstratif hic est, plutôt rare parmi les textes des répons ou des antiennes de l'Office, constitue un topos propre aux épitaphes et aux inscriptions sépulcrales, à l'exemples des suivantes, attribuées au chancelier de Charlemagne :

"Hac magnus colitur Stefanus protomartyr in aula,

Qui fuit egregius meritis et nomine clarus [...]

Ad dexteraque dei stantem super ardua celi $[\ldots]$

Cuius honore pio fecit Ambrosius aedem

Iam precibus veniam mereretur ut illius almis [...]

Utque suum famulum Christus conservet in aevum ${ }^{44}$.

Hic precibus nostris Michael archangelus adsit.

Magnus in arce poli princeps, Christique minister.

Adiuvet ille preces sanctorum semper ubique

Ille suis precibus nos hic conservet ab hoste

Invigilare Dei faciet et laudibus istic ${ }^{45}$.

Hic sit sub pedibus tibi, Marginare, magistri

Iam sacra mundanis temporibus requies ${ }^{46}$..."

Cependant, les inscriptions pouvant elles-mêmes trouver leur source dans les textes liturgiques, il ne faut pas exclure l'hypothèse de l'influence d'un autre office dans la liturgie de saint Martin. Notamment l'office de saint Michel comme il a été mentionné lors de l'analyse musicale, présente outre des analogies mélodiques, des analogies également littéraires et thématiques :

Hic est Michael archangelus princeps milicie angelorum

Cuius honor prestat beneficia populis

Et oratio perducat ad regna celorum.

Archangele Christi per gratiam

Qui meruisti deprecamur per unigenitum dominum nostrum

Ut eripiat nos a laqueo mortis. (CAO 6826)

Ce répons est absent des livres du vieux-romain; on pourrait aussi renvoyer à un répons de saint Jean l'évangéliste Hic est discipulus (CAO 6822) mais surtout à un autre office de tradition pseudo-alcuinienne, celui de saint Vaast, composé à partir d'une Vita écrite par Alcuin vers 800 , le second

42. Voir BHL, t. 2, 825-830 ( ${ }^{\circ}$ 5617-5666).

43. Voir la communication de Cécile Treffort.

44. PL 101, col. 756.

45. PL 101, col. 774.

46. J. MABILLon, Annales, t. II, 284; voir idem t. II, libr. xxvii, 361. 
répons qui commence par cette expression démonstrative Hic est ( $C A O$ 6829). Mais la modélisation du répons sur les formulaires de saint Michel semble une hypothèse d'autant plus probable qu'un lien entre l'hagiographie martinienne et la littérature concernant la dédicace de l'Archange est établi par Grégoire de Tours, qui nous rapporte dans son Livre des vertus de saint Martin (BHL 5618) que le confesseur fut emmené aux cieux par l'archange, avec les autres anges, renforçant la gloire de l'Église et de ses prêtres (R. Ecclesia virtute V. O beatum CAO 6621) ${ }^{47}$. Le verset du répons, écrit à la gloire du dogme de la Trinité ne serait-il pas à mettre en relation avec le Liber sancti Martini de Trinitate que l'on trouve dans les martinelli, parfois placé entre un Sermo de transitu de Grégoire de Tours et des inscriptions?

C'est également du côté des martinelli carolingiens et des homéliaires que des recherches pourraient enrichir le dossier, tant pour repérer d'autres sources hagiographiques et littéraires du répons Hic est comme des autres compositions, et de même, repérer l'ampleur de la diffusion de l'office en Europe ${ }^{48}$.

\section{Réécriture et modifications romaines}

La comparaison littéraire des formulaires romano-franc et vieux-romain semble essentielle au niveau des répons, notamment pour le premier. Visà-vis de cette tradition pourtant bien établie et unanime d'après les plus anciens témoins de la fin du $\mathrm{IX}^{\mathrm{e}}$ et du $\mathrm{X}^{\mathrm{e}}$ siècle, les antiphonaires romains substituent à l'incipit démonstratif $H i c$ est, un incipit qualificatif beaucoup plus courant Beatus. Le démonstratif, peut-être plus valorisant, n'a été réservé que pour quelques pièces de grandes fêtes, par exemple pour saint Jean. En revanche, pour les martyrs comme pour les confesseurs, de nombreux répons commencent simplement par l'expression Beatus suivie du nom du saint. Et justement, le premier répons de saint Martin est un des représentants types de ce groupe les plus représentatifs du $7^{\mathrm{e}}$ mode, à l'exemple du Beatus Valentinus $\left(\mathrm{f}^{\circ} 189 \mathrm{v}^{\circ}\right)$. L'influence d'une messe propre, par exemple la Beatus Martinus, assez répandue dans la péninsule et à Bénévent à la fin de l'époque carolingienne constituerait-elle également un mobile?

L'autre modification apportée à l'incipit concerne le répons $O$ beatum virum, pour éviter probablement la confusion de ces deux pièces dont la mélodie est chantée dans le même mode dans les deux formulaires, et avec le même incipit dans le romano-franc : même type de modification ici, la substitution de $O$ beatum virum par Beatus Martinus.

47. MGH SS rer. Merov., t. 1, c. 5 .

48. M. Heinzelmann et P. BouRgain, L'œuvre de Grégoire de Tours..., p. 273-317. 


\section{La dialectique " Romain/Grégorien " au temps d'Alcuin}

Ces modifications littéraires des témoins vieux-romains m'amènent donc à supposer l'adaptation dans la tradition romaine antique, d'un formulaire romano-franc. En effet, l'origine romaine de l'office semble improbable : le culte liturgique des saints a montré qu'il prenait son essor sur le lieu de sépulture. Pour saint Martin, il est évident que la célébrité du pèlerinage au tombeau de Tours et le rayonnement de la communauté des chanoines de la basilique s'impose comme lieu d'origine de l'office, d'autant que le saint dès l'époque franque reçoit le patronage de la dynastie mérovingienne depuis Clovis au moins, sans parler de la dynastie carolingienne dont les plus illustres représentants ont largement protégé le vénérable sépulcre et son institution ecclésiastique. L'origine épigraphique du premier répons ou si l'on renverse l'hypothèse, l'origine liturgique de l'inscription citée par un Pseudo-Alcuin à la fin du viII ${ }^{\mathrm{e}}$ siècle, place la version romano-franque du texte avant la version romaine. Cela semble plus probable, l'adaptation allant dans le sens d'une réécriture un peu moins circonstanciée et historique. Preuve en est, l'absence du verset implorant la Trinité, pourtant essentiel sur le plan théologique, qui laisse place à Rome à un des célèbres miracles du vivant de saint Martin, celui du globe de feu, rapporté par Sulpice Sévère.

Par comparaison avec les autres traditions liturgiques, ont déduit aisément que si Rome avait un formulaire propre et original, il serait indépendant comme à Milan, à Leon ou à Bénévent. Un libellus ou un autre livre liturgique mixte (franco-romain) a du être le modèle de la présente recension romaine, peut-être bien antérieure aux deux témoins de la seconde moitié du XI ${ }^{\mathrm{e}}$ siècle, ce que supposait déjà le Père Pierre-Marie Gy ${ }^{49}$. Les correspondances littéraires et musicales y sont le signe manifeste d'un apport romano-franc à Rome, ce qui ouvre par ailleurs de nouvelles perspectives au sujet des échanges liturgiques et musicaux entre Rome et les Gaules, l'habitude privilégiant souvent l'apport romain vers les Gaules. C'est néanmoins un mouvement qu'avaient remarqué Dom Jean Claire à propos de l'Office de l'Avent ${ }^{50}$, puis également Philippe Bernard, observateurs d'une contamination romano-franque du répertoire vieux-romain antérieure à sa mise par écrit au XI ${ }^{\mathrm{e}}$ siècle ${ }^{51}$.

\section{Permanence des traditions orales entre le IX $\mathrm{X}^{e}$ siècle et le $\mathrm{XI}^{e}$ siècle}

L'analyse des mélodies correspondantes tant au niveau des répons que des antiennes conduit à l'évidence que les compositions issues des textes de Sulpice Sévère, largement connus dans l'ensemble du monde latin, n'ont pas été transmises à Rome ou à Milan par écrit, mais plus volontiers à une

49. D. P.-M. Gy, « Les répons de l'office nocturne... ».

50. D. J. Claire, "La musique de l'Office de l'Avent ".

51. Ph. Bernard, Du chant romain au grégorien... 
époque où c'était la tradition orale des chantres qui opérait. Souvent, à l'exception de groupes bien caractéristiques ou de certains timbres, les mélodies ne sont pas reprises telles quelles avec leur ornementation, mais sont réadaptées avec les matériaux mélodiques, les tournures ornementales et les idiomes propres aux traditions locales de chant, plus archaïsantes en Italie. Ainsi, on constate une véritable différenciation entre les différents répertoires italiques et romano-francs. Comme le constatait Leo Treitler, ce n'est pas la mélodie en tant que telle et à l'identique que l'on retrouve d'une tradition à l'autre, mais la structuration globale des formules et de la récitation à partir d'un même texte littéraire ${ }^{52}$.

\section{Critique des sources et évolution}

L'instabilité des formulaires pour les versets est lui aussi révélateur de l'ancienneté de la diffusion de l'office en Europe. À la différence d'offices d'origine franque comme saint Maurice, saint Denis, Rustique et Eleuthère ou encore Remi, sans parler des offices du $\mathrm{x}^{\mathrm{e}}$ siècle, on constate une diversité encore plus marquée dans celui de saint Martin, tant au niveau de l'ordre des pièces que surtout, la variabilité du texte des versets, donnant souvent lieu à plus de trois versions distinctes, y compris dans des témoins de provenance peu éloignée géographiquement.

Le formulaire du graduel de Sainte-Cécile d'Albi, que John A. Emerson date des environs de $890^{53}$, que le Père Pierre-Marie Gy n'avait pas remarqué, ne fait que renforcer la diversité de l'ordo. Il pourrait se rattacher à une sorte d'archétype à l'instar de l'antiphonaire de Bamberg, mais il ne concorde pas non plus avec le Compendiensis (Bibliothèque nationale de France lat. 17436), antérieur d'à peine vingt-ans, appartenant pourtant à un milieu culturel et liturgique assez proche.

\section{Formulaire d'Albi}

\begin{tabular}{|c|c|c|c|c|c|c|c|c|c|}
\hline $\mathrm{R}^{54}$ & $*$ & $*$ & $*$ & $*$ & 08 & 09 & 05 & 10 & 11 \\
\hline
\end{tabular}

De même un témoignage supplémentaire de l'office est offert par les martyrologes : en l'occurrence l'éloge suivant ajouté par Usuard aux recensions de Florus et d'Adon : In Galliis, Turonis civitate, natalis beati Martini episcopi et confessoris, cuius vita tantis extitit miraculis gloriosa, ut trium mortuorum meruerit esse suscitator ${ }^{55}$.

La fin de cet éloge se présente effectivement comme une citation soit de la fin du corps du répons, le repetendum ou presque, soit, autre possi-

52. L. TREITLER, "Homer and Gregory..." ".

53. J. A. Emerson et L. Collamore, Albi, B. M. Rochegude ms 44...

54 . Albi, Bibliothèque Municiaple, ms. 44, $\mathrm{f}^{\circ} 121 \mathrm{v}^{\circ}-122$ (le texte est acéphale, manquent les antiennes et les premiers répons des matines). Aux laudes, complètes, s'ajoute un supplément in evangelio (laudes et secondes vêpres probablement).

55. Éd. J. DuBoIs, Martyrologe d'Usuard..., p. 340. 
bilité, une citation d'une antienne des premières vêpres, peu diffusée mais néanmoins ancienne, Martinus trium mortuorum (CAO 3715), qui reprend dans une analogie au Christ ce miracle des trois morts ressuscités, comme symbole de la Trinité bienfaisante. Ce témoignage d'Usuard remontre d'après Jacques Dubois vers 870, d'après la dédicace à Charles le Chauve de la seconde recension de son martyrologe.

Pour l'office, le corpus martinien semble bien s'élaborer avant le $\mathrm{IX}^{\mathrm{e}}$ siècle. Son insertion dans le graduel d'Albi non pas entre les fêtes de saint Michel et de sainte Cécile mais après le commun, témoigne de la diffusion progressive de l'historia dans les livres liturgiques en Gaule et dans l'ensemble des églises de l'Empire. Mais les témoins, qui manifestement prennent un certain temps, quelques décennies, pour instituer un nouveau formulaire, ne peuvent en fait que borner un terminus ante quem. Dans cette perspective, l'absence de l'office dans le Liber de ordine antiphonarii d'Amalaire de Metz (vers 830) n'est absolument pas rédhibitoire. Si le Père Pierre-Marie Gy considère que l'office arrive à la chapelle palatine avant le pèlerinage de Louis le Pieux à Tours le 11 novembre 818 ou même de Charlemagne en $800^{56}$, je replacerais volontiers la composition bien avant Fridugise et Alcuin, non seulement à cause du Sermo ou de la Laudatio, de l'analyse comparée des textes et des mélodies romaines et grégoriennes, mais aussi grâce à l'état de la tradition ambrosienne et bénéventaine et à l'analogie avec l'office romain de saint Michel, du vieux-fonds (fin $\mathrm{VII}^{\mathrm{e}}$-VIII ${ }^{\mathrm{e}}$ siècle).

De même il pourrait s'avérer pour certains formulaires de la messe, par la proximité structurelle et stylistique des chants modelés sur des pièces du commun des confesseurs, qu'ils soient antérieurs au $\mathrm{x}^{\mathrm{e}}$ siècle et que leur diffusion ait pu se faire à l'exemple de l'office, dans le cours du IX ${ }^{\mathrm{e}}$ siècle.

Il paraît ainsi plus probable qu'Alcuin, arrivé en " étranger " au monastère Saint-Martin, n'ait pas à la différence d'un Hilduin ou d'un Hincmar, restauré et amplifié un culte, mais simplement témoigné d'une tradition liturgique et musicale déjà bien établie, peut-être depuis que les souverains carolingiens, œuvrant à la romanisation des traditions franques, sont intervenus dans le gouvernement et la protection de ce haut lieu du royaume dans la seconde moitié du viII ${ }^{\mathrm{e}}$ siècle.

\section{Alcuin et son temps : un témoignage essentiel pour l'histoire de la musique}

Certes non exclusive dans la diffusion d'un culte et de répertoires musicaux concomitants, liés à un saint qui dès les $\mathrm{VI}^{\mathrm{e}}$ et $\mathrm{VII}^{\mathrm{e}}$ siècles dépasse largement les cadres des royaumes Francs, le rôle de la métropole de Tours puis du célèbre chancelier carolingien sont loin d'être négligeables dans la transformation d'un culte liturgique local en un culte quasiment universel :

56. D. P.-M. GY, « Les répons de l'office nocturne... », p. 223. 
l'adoption par la Ville éternelle de l'office grégorien élaboré à Tours et dans les sphères carolingiennes à la fin du VIII ${ }^{\mathrm{e}}$ siècle représente une véritable consécration pour le vénérable miles Christi. Mais surtout, sur un plan d'esthétique musicale, notamment pour comprendre l'évolution progressive des chants dans les églises latines à l'époque carolingienne où romanisation et hybridation s'imposent comme concepts majeurs, ce fait donne un contre-exemple tout à fait remarquable de l'un des mécanismes des plus complexes de l'histoire de la musique et donne une précieuse contribution à ce que Willi Apel considérait comme une des " questions centrales " du chant grégorien dans sa dialectique entre Rome et les Gaules ${ }^{57}$. Le corpus martinien montre comment vers 750-800 la réforme de traditions de chant s'effectue dans un contexte de tradition orale, non pas par imitation exacte d'une mélodie, qu'elle fût ou non appuyée par je ne sais quel archétype carolingien avec notation ${ }^{58}$, mais implique plutôt à partir d'un texte littéraire commun, une adaptation de la cantilena qui maintient des usages antérieurs, différenciés dans leur ornementation, leur modalité et leur interprétation. Le chant est bien demeuré une tradition vivante dans le premier millénaire.

57. W. APEL, "The Central Problem of Gregorian Chant ".

58. Les positions de Kenneth Levy peuvent-être acceptables quant à l'inversion du sens des échanges entre le vieux-romain et le grégorien, ne nécessitant aucunement le maintien de l'hypothèse d'un archétype carolingien écrit du graduel, ni une transmission mécanique des mélodies. cf. K. LEVY, « Gregorian chant and the Romans ». 


\section{RESUME}

Même si on lui attribue un traité De Musica aujourd'hui perdu, Alcuin ne s'associe pas aussi directement que ses successeurs tels Amalaire de Metz ou Hilduin de Saint-Denis à la composition musicale et même à l'histoire de la musique à l'époque carolingienne : le magister s'est davantage illustré dans le domaine de la stricte liturgie.

Il apparaît néanmoins qu'un détail de son œuvre d'hagiographe, notamment un opuscule périphérique relatif au saint patron du monastère auquel Charlemagne l'invite à prendre la direction à la fin de sa vie, et dont l'attribution est discutée, permet de définir une perspective intéressante quant à la datation de l'office de saint Martin et surtout, quant à l'une des " questions centrales " du chant grégorien (Willi Apel), à savoir la romanisation des traditions de chant liturgique dans l'Empire au $\mathrm{VIII}^{\mathrm{e}}$ siècle et l'influence des monarques carolingiens dans la dialectique complexe entre Rome et les Gaules en ce domaine dans le haut Moyen Âge.

\section{ABSTRACT}

Although a treatise De Musica now lost could have been written by him, Alcuin is not so directly associated with musical composition and not easily involved for musicologists in History of music in the Carolingian era, as could be authors from the next generation, for example Amalaire of Metz or Hilduin. The magister wrote mainly and first of all works strictly devoted to Liturgy.

Nevertheless, one of his hagiographical works concerning the patron saint of the major Monastery where Charlemagne placed him abbot at the end of his life, yet discussed for its authenticity, offers mostly important points about datation of the St. Martin's Office and overall, gives an interessant point of view concerning what Willy Apel called "the central problem of Gregorian chant": romanisation of liturgical chant traditions through the Empire during the $8^{\text {th }}$ century and the role of Carolingian kings in the complex dialectic between Rome and Gaul during High Middle Ages. 
